\title{
EDITORIAL
}

\section{IN THE MAY 2010 ISSUE OF CLINICS}

\author{
Mauricio Rocha e Silva \\ doi: 10.1590/S1807-59322010000500001
}

In this issue of CLINICS, we open a new section entitled "Bringing Ideas Together" in which experienced authors will contribute a measure of speculative thought on apparently unrelated themes in medical practice. This section will only occur when a sufficiently interesting article is offered to us. In this issue, a paper by Pereira et al. on the imbalance between thyroid hormones and the dopaminergic system as possible central central factors in the pathophysiology of restless legs syndrome is offered for discussion. Comments, letters and editorials are welcome, subject of course to peer evaluation. In this issue we publish ten original clinical science papers, one review and three case reports.

Otsuki et al. note that proprietary sevoflurane is diluted in water, whereas a generic sevoflurane (Sevocris) is produced with propylene glycol as a stabilizing additive. They investigated whether the original and generic sevoflurane preparations differed in terms of their minimum alveolar concentration values and hemodynamic effects in aporcine experimental model: their results indicate that the propylene glycol added sevoflurane can be used as safely as the water diluted sevoflurane, regarding hemodynamic and pulmonary effects.

Senne et al. assessed the influence of Nd: YAG laser unilateral posterior capsulotomy on visual acuity and perception of difficulties with vision-related daily life activities through an interventional survey with 48 patients aged between 40 and 80 years, with uni- or bilateral pseudophakia, posterior capsule opacification and visual acuity equal to or less than 0.30 (logMAR) in one eye. All the patients were submitted to posterior capsulotomy by Neodymium: Yttrium-Aluminium-Garnet (Nd: YAG) laser, resulting in a significant improvement of visual acuity in the capsulotomized eye and, according to the subjects' perception, a reduction of difficulty in most visiondependent daily activities.

Hospital das Clínicas, Faculdade de Medicina da Universidade de São Paulo - São Paulo/SP, Brazil.

mrsilva36@hcnet.usp.br
Heldwein et al. endeavored to identify the most cited articles in general surgery published by Brazilian authors between 1970 and 2010, over which the Brazilian output of surgical research increased 21-fold. Out of 1,713 articles, they identified 9 classics (more than 100 citations received), the majority of which were the result of international cooperation.

Takara et al. compared the measurements of spirometric peak expiratory flow (PEF) from five different PEF meters and to determine whether their values are in agreement. Comparisons were performed in 68 healthy, sedentary and insufficiently active subjects, aged from 19 to 40 years old, using Air Zone ${ }^{\circledR}$, Assess ${ }^{\circledR}$, Galemed ${ }^{\circledR}$, Personal Best ${ }^{\circledR}$ and Vitalograph ${ }^{\circledR}$ peak flow meters. No agreement was found between the spirometric values and the five PEF meters. The results suggest that Galemed ${ }^{\circledR}$ recorded values may have possibly underestimated true values and thereby leading to an intervention that, in fact, would not be necessary, while Air Zone ${ }^{\circledR}$ meter values may have been overestimates covering up the need for intervention. They indicate that these points must be taken into account when interpreting the readings from both devices for younger people. Theey also stress the attention required when directly comparing different types of PEF meters recorded values.

Kargili et al. note that the association between polycystic ovarian syndrome and increased cardiovascular disease risk is still a controversial issue. Consequently they conducted a study to determine whether there is a relationship between sleep blood pressure pattern disturbances and polycystic ovarian syndrome in 168 young women aged $26 \pm 5$ years. Authors found that a non-dipping blood pressure pattern has a high prevalence in polycystic ovarian syndrome patients even if they are young and non-obese.

Lin et al. examined risks, rates of readmission and their predictors 14 days, one year, and five years after discharge for the psychiatric population in Taiwan through a claim data-based prospective study with 44,237 firsttime hospitalized psychiatric patients discharged in 2000, and followed for up to 5 years of discharge. Cumulative 
incidence (CI) and incidence density (ID) of readmission were calculated for various follow-up periods after discharge; and Cox-proportional hazard models were conducted to identify the significant predictors. They found that significant predictors for psychiatric readmission in 14 days to 5 years after discharge were essentially the same (male gender, length of hospital stay $>15$ days, economically poor) except patient's age and hospital accreditation level. This study also highlighted the importance of socioeconomic factors in the prediction of readmission. In contrast young adults (20-39 years) were significantly associated with increased risks of $<1$-year and $<5$-year readmissions, but not so for $<14$-day readmission.

Eren et al. present special clinical and laboratory features of 294 cases of mushroom poisoning admitted to the Pediatric and Adult Emergency, Internal Medicine and ICU Departments of Cumhuriyet University Hospital. Nearly $60 \%$ had consumed the mushrooms in the early summer. Almost all patients (98\%) developed early symptoms, one third of them being positive within two hours of ingestion. The most common occurrences affected the gastrointestinal system. Patients were discharged after 1-10 days, but 3 died from fulminating hepatic failure. Authors emphasize the need for education of the public about consuming mushrooms and health personnel for early treatment and transfer appropriate hospitals.

Yuan SM et al. studied the relation between congenital bicuspid aortic valve aortic dilation in 241 consecutive patients over a 5 year period by comparing them to a matched group of tricuspid aortic valve patients. The bicuspid aortic valve patients were younger with male gender predominance. Most of them were stenotic, regurgitant, or combined, with only $19(<8 \%)$ normally functioning. The dimensions of the aortic root, sinotubular, and ascending aorta were larger in both groups, but significantly smaller in the bicuspid aortic valve than in tricuspid aortic valve patients, and much smaller in patients receiving statin therapy than those without in both groups. Moreover, the aortic dilation in the bicuspid group was significantly age-related.

Kanashiro et al. describe the results of 117 nephrectomies performed using a subcostal mini -incision. Surgical time, warm and cold ischemia time, intra-operatory complications, time for hospital discharge, presence of infection, bleeding, the need for a second operation, or death were analyzed. Eventual loss of donor renal function was followed by doses of serum creatinine and proteinuria. They conclude that the use of a subcostal mini-incision is safe and similar to conventional techniques previously described in the literature.

Scanavino et al. investigated sexual dysfunction symptoms in patients with HIV, which have not been fully described in Brazil through an anonymously completed, self-responsive inquiry applied to a population sample in 18 Brazilian big cities. Answers by AIDS patients (75) were compared with control group (150). In the female sample, AIDS was associated with "sex inactivity in the last 12 months", and "do not keep maintain sexual arousal until the end of sex act" $(\mathrm{P}<0.05)$ after adjusting for race, and thyroid disease. Men reporting AIDS showed more difficulty to develop sexual response (they need more help of the partner to begin the sex act, they have longer foreplay than they wish, more do not keep the sexual desire until the end of the sex, a longer than desired time to ejaculation. Authors claim that their results support the hypothesis that sexual dysfunctions are associated factors to HIV/AIDS, independently of others factors.

We publish a review by Scorza et al. on thalamic nuclear abnormalities as a contributory factor in sudden cardiac deaths among patients with schizophrenia. We also publish 3 case reports 